\title{
Herbal Medicine for Patients with Cognitive Impairment: An Observational Study
}

\author{
Yujin Choi (D) \\ Ae-Ran Kim (D) ${ }^{2}$ \\ Ji-Yoon Lee ${ }^{3}$ \\ Hae Sook Kim (iD) ${ }^{3}$ \\ Changsop Yang $\mathbb{D}^{\prime}$ \\ Jae Kwang Kim ${ }^{4}$ \\ Younghoon Go $\mathbb{D}^{4}$ \\ In Chul Jung $\mathbb{D}^{3}$
}

'KM Science Research Division, Korea Institute of Oriental Medicine, Daejeon, Republic of Korea; ${ }^{2}$ R\&D Strategy Division, Korea Institute of Oriental Medicine, Daejeon, Republic of Korea; ${ }^{3}$ Department of Neuropsychiatry, College of Korean Medicine, Daejeon University, Daejeon, Republic of Korea; ${ }^{4} \mathrm{KM}$ Application Center, Korea Institute of Oriental Medicine, Daegu, Republic of Korea
Correspondence: In Chul Jung

Department of Neuropsychiatry, College of Korean Medicine, Daejeon University,

75 Daedeok-daero I76beon-gil, Seo-gu,

Daejeon, Republic of Korea

Tel +82-42-470-9129

Fax +82-42-470-9005

Email npjeong@dju.kr
Purpose: The potential effects of herbal medicine for patients with cognitive disorders have been reported in various human and animal studies. This study aimed to explore the effect of herbal medicine treatment according to the Korean Medicine (KM) pattern identification for patients with mild cognitive impairment and early dementia.

Patients and Methods: Twenty patients with mild cognitive impairment or mild dementia who planned to receive herbal medicine treatment were enrolled. Herbal formulae were prescribed based on the KM pattern for 12-24 weeks. Seoul Neuropsychological Screening Battery II (SNSB-II) and Montreal Cognitive Assessment (MoCA) were assessed at the baseline, after 12 weeks, and after 24 weeks (Trial registration: cris.nih.go.kr, KCT0004799). Results: Herbal medicine products, including Yukmijihwang-tang, Samhwangsasim-tang, Palmul-tang, Banhasasim-tang, and Yukgunja-tang, were prescribed to the patients. Among the SNSB-II five cognitive function domains, the T scores for language, visuospatial function, memory, and frontal/executive function increased over time. The MoCA score also improved following the treatment (mean difference 4.23 [95\% CI: 2.60, 5.86], p < 0.0001 at 12-week follow-up compared to the baseline). Considering the KM pattern scores, phlegm-dampness and fire-heat scores tended to improve after the treatment. No serious adverse events related to the intervention were reported.

Conclusion: The potential effect of herbal medicine formulae products on improving cognitive functions in patients with cognitive impairment was observed. Further research is needed to objectify the KM pattern identification process and evaluate the KM patternrelated signs and symptoms.

Keywords: mild cognitive impairment, mild dementia, herbal medicine, Korean medicine, traditional east Asian medicine, observational study

\section{Introduction}

Neurocognitive disorders (NCD) are degenerative disorders with main symptoms of deficits in various cognitive functions. ${ }^{1,2}$ Mild and major NCDs are distinguished according to the maintenance of activities of daily living. The deficits can have massive impacts on the quality of life for both patients and caregivers. Recently, the potential effects of herbal medicine, which are widely used for age-related degenerative disease in east Asian countries, have been reported for patients with cognitive disorders. ${ }^{3,4}$ A systematic review summarized that Chinese herbal medicine improved the cognitive scores of patients with mild cognitive impairment (MCI). ${ }^{5}$ Another review reported that herbal medicine alleviated behavioral and psychological symptoms of patients with dementia. ${ }^{6}$ Several observational studies of herbal medicine as treatment for cognitive disorders have also been reported, including Dangguijagyag-san ${ }^{7}$ and Ninjinyoei-to. ${ }^{8}$ 
In Traditional East Asian Medicine (TEAM), pattern identification, also called syndrome differentiation, is the core clinical decision process in clinical practice. ${ }^{9,10}$ Traditionally, practitioners diagnose the Traditional Chinese Medicine (TCM) or Korean Medicine (KM) patients' patterns after the professional and subjective pattern identification process of observation, listening, questioning, and pulse analysis. In recent studies, efforts have been made to measure the TCM or KM pattern objectively in patients with cognitive impairment. ${ }^{11,12}$ Specifically, in a real-world multicenter controlled clinical study conducted in China, sequential therapy based on the evolvement of patterns (STEP) regimen was applied for patients with Alzheimer's Disease (AD) $)^{13,14}$ and the authors concluded that early $\mathrm{AD}$ initiates from kidney deficiency, and as the disease progress, phlegmdampness, blood stasis, and fire-heat could occur pathologically. According to the sequential patterns of $\mathrm{AD}$ patients, herbal medicine demonstrated beneficial effects in maintaining cognitive stability in patients with $\mathrm{AD}$ after the 2-years of follow-up.

In Korea, a pattern identification tool for cognitive disorders (PIT-C) has been developed. ${ }^{15,16}$ It does not distinguish the pattern of cognitive disorders according to the stage of the disease. Instead, PIT-C comprises two deficiency patterns, qi-deficiency and yin-deficiency, and two excess patterns, phlegm-dampness and heat-fire. The Qi-deficiency score measures the symptoms of fatigue and lack of energy. The Yin-deficiency score measures the symptoms of hot flushes and ringing in the ears. Phlegmdampness score is composed of the symptoms of a feeling of heaviness in the head and digestive issues. The fire-heat score is composed of the symptoms of being angered easily, irritability, or aggression.

Seo et al generated a list of licensed herbal medicine products in Korea that are expected to have potential effects on neurocognitive impairment. ${ }^{17}$ Licensed herbal medicine products are produced in good manufacturing practice (GMP) according to the Korean Herbal Pharmacopoeia (KHP). To select the treatment candidates, individual herbs' effects on neuroinflammation (nitric oxide release inhibition), neuronal cell proliferation (NE-4C cell viability), and energy production target (PDH activity in AD293 cell) were assessed using in vitro assays. ${ }^{18-20}$ The final list of herbal medicine products for patients with neurocognitive disorders was obtained by combining the results of the screening test and the recommendation of experts. ${ }^{17}$
This study aimed to explore the effect of herbal medicine treatment according to the KM pattern identification on cognitive functions and $\mathrm{KM}$ pattern scores. Also, PIT- $\mathrm{C}^{15}$ was applied in KM pattern identification and determination of prescription formula for patients with neurocognitive disorders.

\section{Patients and Methods Trial Design and Ethics Approval}

Patients with neurocognitive disorders who visited the Daejeon Korean Medicine Hospital of Daejeon University were asked written consents, then enrolled in this prospective, observational study. Enrolled patients were treated and managed by qualified clinicians, mainly using herbal medicine. Clinical outcomes were measured at baseline, after 12 weeks, and after 24 weeks. This observational study protocol was approved by the Institutional Review Board of Daejeon University Daejeon Oriental Hospital (DJDSKH-18-BM-21). The approved protocol was prospectively registered at the clinical research information service (cris.nih.go.kr, Registration no. KCT0004799). This study was conducted in accordance with the Declaration of Helsinki.

\section{Participant Flow}

A total of 20 patients with neurocognitive disorders were enrolled in the study. Six patients received SHS and five patients received PMT. For three patients, herbal medicine prescriptions were revised to another formula at the follow-up visits (Figure 1). The duration of administration, prescribed herbal medicine, and compliance in each patient are presented in Table S1. Compliance on taking herbal medicine was greater than $70 \%$ for all patients.

\section{Participants}

Twenty patients with MCI or mild dementia who planned to receive herbal medicine treatment were registered for the study. A hospital in Daejeon, Korea, recruited patients through a notice on the bulletin board in the hospital. Eligible patients were enrolled from 29 July 2019 to 29 May 2020. The follow-up observation of the last participant was completed on 7 September 2020.

The inclusion criteria were as follows:

1. between 45 and 84 years of age; 


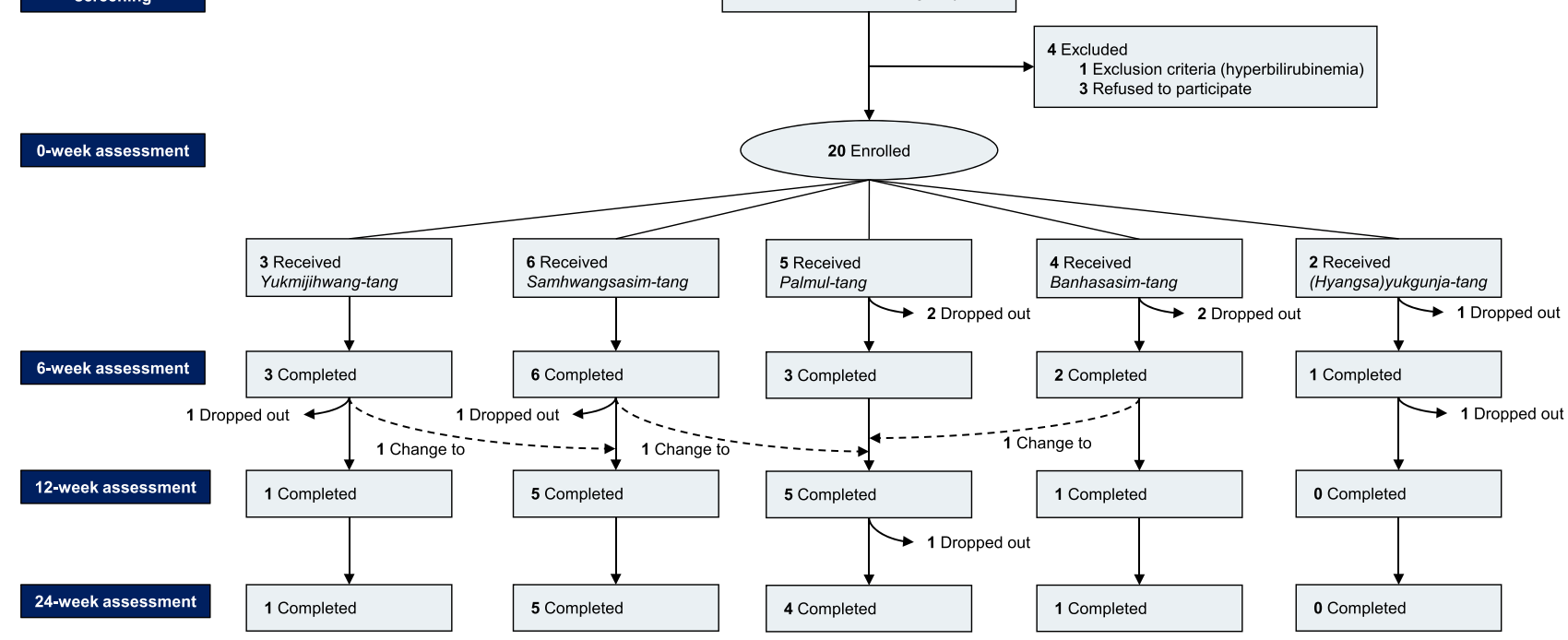

Figure I Flow chart.

2. diagnosis of major or mild neurocognitive impairment based on the criteria of Diagnostic and Statistical Manual of Mental Disorders-5 (DSM-5);

3. Clinical Dementia Rating $(\mathrm{CDR})=0.5$;

4. Global Deterioration Scale (GDS) score 2-5;

5. Montreal Cognitive Assessment (MoCA) score $<23$;

6. capable of understanding the contents of the questionnaire directly or through a guardian; and

7. willingness to participate and those who voluntarily signed the informed consent form.

The exclusion criteria were as follows:

1. history of brain damage or mental retardation;

2. history of Huntington's disease, normal hydrocephalus, or brain tumor;

3. uncontrolled gastrointestinal, endocrine, or cardiovascular diseases;

4. uncontrolled diabetics;

5. severe liver disease, or kidney disease;

6. anemia, hypothyroidism, vitamin deficiency, or malignant disease;

7. severe unstable medical conditions;

8. history of major psychiatric disorders (schizophrenia, delusional disorder, depressive disorder, bipolar disorder, alcohol or substance use disorder);

9. experience of participating in other clinical trials with interventions in the last 4 weeks

10. women of childbearing age;
11. unable to understand the consent form or difficulty in proceeding with the research due to mental retardation, emotional, or intellectual impairments;

12. blindness, hearing loss, or severe speech impairment;

13. not appropriate according to the judgment of the researcher;

\section{Herbal Medicine Treatment}

A list of herbal medicine products generated in a previous study ${ }^{17}$ was mainly used. Among the list of herbal medicine products, the clinicians made a decision on the personalized herbal medicine formulas for each patient based on the KM pattern identification. KM pattern scores of Qi-deficiency, Yin-deficiency, Phlegmdampness, and Fire-heat were measured by the pattern identification tool for cognitive disorders (PIT-C), ${ }^{15,16}$ and clinical impressions were comprehensively considered for the selection of herbal medicine formula. The symptoms and signs of the four patterns in patients with cognitive disorder are listed in Table 1. For patients with high Yin-deficiency score, Yukmijihwang-tang (YMJ; Liu-wei-di-huang-tang in Chinese; Lokumijio-to in Japanese) was selected. For patients with high Fireheat scores, Samhwangsasim-tang (SHS; San-huang-xiexin-tang in Chinese; Sano-shashin-to in Japanese) was selected. For patients with high Phlegm-dampness score, Banhasasim-tang (BHS; Ban-xia-xie-xin-tang in Chinese; Hangeshashin-to in Japanese) was selected. 
Table I Symptoms and Signs of the Four Korean Medicine Patterns in Patients with Cognitive Disorders

\begin{tabular}{|c|c|c|}
\hline Categories & Symptoms and Signs & Patterns \\
\hline $\begin{array}{l}\text { Behavioral/ } \\
\text { Psychological } \\
\text { Symptoms }\end{array}$ & $\begin{array}{l}\text { Slow to react } \\
\text { Lethargic } \\
\text { Easily angry or irritable } \\
\text { Aggressive behavior }\end{array}$ & $\begin{array}{l}\text { Qi-deficiency } \\
\text { Qi-deficiency } \\
\text { Fire-heat } \\
\text { Fire-heat }\end{array}$ \\
\hline Body Energy/Shape & $\begin{array}{l}\text { Easily get tired } \\
\text { Weak voice } \\
\text { Emaciated }\end{array}$ & $\begin{array}{l}\text { Qi-deficiency } \\
\text { Qi-deficiency } \\
\text { Yin-deficiency }\end{array}$ \\
\hline Cold/Heat & $\begin{array}{l}\text { Hot flush } \\
\text { Prefers to drink cool } \\
\text { water }\end{array}$ & $\begin{array}{l}\text { Yin-deficiency } \\
\text { Fire-heat }\end{array}$ \\
\hline Head & $\begin{array}{l}\text { Feels heavy in the head } \\
\text { Buzzing in the ears }\end{array}$ & $\begin{array}{l}\text { Phlegm-dampness } \\
\text { Yin-deficiency }\end{array}$ \\
\hline Face & $\begin{array}{l}\text { Bloodshot eyes } \\
\text { Excessive sputum } \\
\text { Dry mouth }\end{array}$ & $\begin{array}{l}\text { Fire-heat } \\
\text { Phlegm-dampness } \\
\text { Yin-deficiency }\end{array}$ \\
\hline Chest & $\begin{array}{l}\text { Chest congestion } \\
\text { Shortness of breath on } \\
\text { exertion }\end{array}$ & $\begin{array}{l}\text { Phlegm-dampness } \\
\text { Qi-deficiency }\end{array}$ \\
\hline Digestion & $\begin{array}{l}\text { Feels nauseous } \\
\text { Feels bloated }\end{array}$ & $\begin{array}{l}\text { Phlegm-dampness } \\
\text { Phlegm-dampness }\end{array}$ \\
\hline Urine/Stool & $\begin{array}{l}\text { Dark urine } \\
\text { Hard stool }\end{array}$ & $\begin{array}{l}\text { Fire-heat } \\
\text { Yin-deficiency }\end{array}$ \\
\hline Tongue diagnosis* & $\begin{array}{l}\text { Pink tongue }+ \text { thin } \\
\text { white coating } \\
\text { Red tongue }+ \text { little } \\
\text { amount of coating } \\
\text { Pale swollen tongue }+ \\
\text { thick white coating } \\
\text { Red tongue }+ \text { yellow } \\
\text { coating }\end{array}$ & $\begin{array}{l}\text { Qi-deficiency } \\
\text { Yin-deficiency } \\
\text { Phlegm-dampness } \\
\text { Fire-heat }\end{array}$ \\
\hline Pulse diagnosis* & $\begin{array}{l}\text { Fine and weak pulse } \\
\text { (+sunken pulse) } \\
\text { Fine pulse (+rapid pulse) } \\
\text { Slippery pulse } \\
\text { String-like pulse (+rapid } \\
\text { pulse) }\end{array}$ & $\begin{array}{l}\text { Qi-deficiency } \\
\text { Yin-deficiency } \\
\text { Phlegm-dampness } \\
\text { Fire-heat }\end{array}$ \\
\hline
\end{tabular}

Notes: The symptoms and signs of the four patterns presented are based on the Pattern Identification Tool for Cognitive Disorder (PIT-C) version 2. I. Modified from Lee JY, Kim $\mathrm{H}$, Seo YK, Kang HW, Kang WC, Jung IC. A research to evaluate the reliability and validity of pattern identifications tool for cognitive disorder: a clinical study protocol. J Orient Neuropsychiatry. 2018;29(4):255-266. Copyright (c) 2018 by The Korean Society of Oriental Neuropsychiatry. All rights reserved. CC This is an open access article distributed under the terms of the Creative Commons Attribution Non-Commercial License (http://creativecommons.org/licenses/by-nc/4.0). ${ }^{15}$ Each pattern includes five items of symptoms and signs, and each item scores from 0 to 4 (0, Not at all; I, Slightly; 2, moderately; 3, Very; and 4, Extremely). *Tongue and pulse diagnosis are not included in the pattern score calculation.
For patients with high Qi-deficiency scores, Yukgunjatang (YGJ; Liu-jun-zi-tang in Chinese; Ikkunshi-to in Japanese) was selected, and Hyangsayukgunja-tang (Xiang-sha-liu-jun-zi-tang in Chinese; Kosharikkunshito in Japanese) was also considered for patients with Qideficiency and digestive problems. For patients with high Qi-deficiency score and tendency of Blooddeficiency, Palmul-tang (PMT; Bawu-tang in Chinese; Hachimotsu-to in Japanese) was prescribed.

The herbal medicine product (extract granules) manufactured by the pharmaceutical company according to the Korean Herbal Pharmacopoeia (KHP) were used. The ingredients and composition of each herbal medicine formula are presented in Table 2. Moreover, the detailed production methods and quality standards of each herbal medicine formula extract are presented in Supplement 1. The granules were administrated three times a day for 24 weeks.

\section{Outcome Measurement}

The Seoul neuropsychological screening battery II (SNSBII) is composed of various cognitive tests for five cognitive function domains; attention, language, visuospatial function, memory, and frontal/executive function. ${ }^{21,22}$ The digit span test (DST) for attention domain; Boston naming test (BNT) for language domain; Clock drawing test (CDT) for visuospatial function domain; Rey complex figure test (RCFT) for visual memory, Seoul verbal learning test for verbal memory; and Color word Stroop test (CWST) for frontal/executive function domains are the representative tests included in the battery. Additionally, the Montreal Cognitive Assessment (MoCA), and short version of the geriatric depression scale (SGDepS) were carried out. Outcomes were measured at the baseline, after 12 weeks, and after 24 weeks. The MoCA was additionally conducted after six weeks. Integrative Medicine Patient Satisfaction Scale (IMPSS) ${ }^{23}$ was measured after 12 and 24 weeks. Adverse events following the treatment were carefully documented throughout the study. Further, complete blood count, liver and kidney function tests were conducted before and after the treatment for safety evaluation.

\section{Statistical Methods}

Continuous variables are presented as mean \pm standard deviation and categorical variables are presented as a frequency $(\%)$. The linear mixed model for repeated 
Table 2 The Herbal Ingredients and Composition Ratio of Formulae

\begin{tabular}{|c|c|c|c|c|}
\hline Formula Name & Herbal Name & Source Species & Parts Used & $\begin{array}{c}\text { Composition } \\
\text { Ratio }\end{array}$ \\
\hline Yukmijihwang-tang & $\begin{array}{c}\text { Rehmanniae Radix Preparata } \\
\text { Dioscoreae Rhizoma } \\
\text { Corni Fructus } \\
\text { Poria Sclerotium } \\
\text { Moutan Radicis Cortex } \\
\text { Alismatis Rhizoma }\end{array}$ & $\begin{array}{c}\text { Rehmannia glutinosa (Gaertn.) DC. } \\
\text { Dioscorea polystachya Turcz. } \\
\text { Cornus officinalis Siebold \& Zucc. } \\
\text { Poria cocos Wolf } \\
\text { Paeonia } \times \text { suffruticosa Andrews } \\
\text { Alisma plantago-aquatica subsp. orientale (Sam.) } \\
\text { Sam. }\end{array}$ & $\begin{array}{l}\text { Root } \\
\text { Rhizome } \\
\text { Fruit } \\
\text { Sclerotium } \\
\text { Rhizodermis } \\
\text { Rhizome }\end{array}$ & $\begin{array}{l}28.6 \% \\
14.3 \% \\
14.3 \% \\
14.3 \% \\
14.3 \% \\
14.3 \%\end{array}$ \\
\hline $\begin{array}{l}\text { Samhwangsasim- } \\
\text { tang }\end{array}$ & $\begin{array}{c}\text { Rhei Radix et Rhizoma } \\
\text { Coptidis Rhizoma } \\
\text { Scutellariae Radix }\end{array}$ & $\begin{array}{c}\text { Rheum palmatum L. } \\
\text { Coptis japonica (Thunb.) Makino } \\
\text { Scutellaria baicalensis Georgi }\end{array}$ & $\begin{array}{c}\text { Root and rhizome } \\
\text { Rhizome } \\
\text { Root }\end{array}$ & $\begin{array}{l}40.1 \% \\
29.9 \% \\
29.9 \%\end{array}$ \\
\hline Palmul-tang & $\begin{array}{c}\text { Ginseng Radix } \\
\text { Atractylodis Rhizoma Alba } \\
\text { Poria Sclerotium } \\
\text { Glycyrrhizae Radix et } \\
\text { Rhizoma } \\
\text { Angelicae Gigantis Radix } \\
\text { Cnidii Rhizoma } \\
\text { Paeoniae Radix } \\
\text { Rehmanniae Radix Preparata }\end{array}$ & $\begin{array}{c}\text { Panax ginseng C.A.Mey. } \\
\text { Atractylodes macrocephala Koidz. } \\
\text { Poria cocos Wolf } \\
\text { Glycyrrhiza uralensis Fisch. ex DC. } \\
\text { Angelica gigas Nakai } \\
\text { Ligusticum officinale (Makino) Kitag. } \\
\text { Paeonia lactiflora Pall. } \\
\text { Rehmannia glutinosa (Gaertn.) DC. }\end{array}$ & $\begin{array}{c}\text { Root } \\
\text { Rhizome } \\
\text { Sclerotium } \\
\text { Root and rhizome } \\
\text { Root } \\
\text { Rhizome } \\
\text { Root } \\
\text { Root }\end{array}$ & $\begin{array}{l}12.5 \% \\
12.5 \% \\
12.5 \% \\
12.5 \% \\
12.5 \% \\
12.5 \% \\
12.5 \% \\
12.5 \%\end{array}$ \\
\hline Banhasasim-tang & $\begin{array}{c}\text { Pinelliae Tuber } \\
\text { Scutellariae Radix } \\
\text { Ginseng Radix } \\
\text { Glycyrrhizae Radix et } \\
\text { Rhizoma } \\
\text { Zingiberis Rhizoma } \\
\text { Coptidis Rhizoma } \\
\text { Zizyphi Fructus }\end{array}$ & $\begin{array}{l}\text { Pinellia ternata (Thunb.) Makino } \\
\text { Scutellaria baicalensis Georgi } \\
\text { Panax ginseng C.A.Mey. } \\
\text { Glycyrrhiza uralensis Fisch. ex DC. } \\
\text { Zingiber officinale Roscoe } \\
\text { Coptis japonica (Thunb.) Makino } \\
\text { Ziziphus jujuba Mill. }\end{array}$ & $\begin{array}{c}\text { Tuber } \\
\text { Root } \\
\text { Root } \\
\text { Root and rhizome } \\
\text { Rhizome } \\
\text { Rhizome } \\
\text { Fruit }\end{array}$ & $\begin{array}{l}24.5 \% \\
14.6 \% \\
14.6 \% \\
14.6 \% \\
\\
12.2 \% \\
4.8 \% \\
14.6 \%\end{array}$ \\
\hline Yukgunja-tang & $\begin{array}{c}\text { Atractylodis Rhizoma Alba } \\
\text { Poria Sclerotium } \\
\text { Pinelliae Tuber } \\
\text { Citri Unshius Pericarpium } \\
\text { Ginseng Radix } \\
\text { Glycyrrhizae Radix et } \\
\text { Rhizoma } \\
\text { Zingiberis Rhizoma Recens } \\
\text { Zizyphi Fructus }\end{array}$ & $\begin{array}{c}\text { Atractylodes macrocephala Koidz. } \\
\text { Poria cocos Wolf } \\
\text { Pinellia ternata (Thunb.) Makino } \\
\text { Citrus deliciosa Ten. } \\
\text { Panax ginseng C.A.Mey. } \\
\text { Glycyrrhiza uralensis Fisch. ex DC. } \\
\text { Zingiber officinale Roscoe } \\
\text { Ziziphus jujuba Mill. }\end{array}$ & $\begin{array}{c}\text { Rhizome } \\
\text { Sclerotium } \\
\text { Tuber } \\
\text { Pericarpium } \\
\text { Root } \\
\text { Root and rhizome } \\
\text { Rhizoma } \\
\text { Fruit }\end{array}$ & $\begin{array}{l}15.7 \% \\
15.7 \% \\
15.7 \% \\
15.7 \% \\
15.7 \% \\
5.9 \% \\
\\
7.9 \% \\
7.9 \%\end{array}$ \\
\hline
\end{tabular}

measures was used to compare clinical outcomes over time. The least-squares mean and standard deviation over time were calculated. Additionally, mean differences and $95 \%$ confidence intervals of week 6 , week 12, and week 24 compared to baseline scores (week 0) were estimated. Tukey's method was used to adjust the p-value for the multiple comparisons. The minimal clinically important difference (MCID) and minimum detectable change (MDC) were considered for the interpretations of the results. For the SNSB-II five cognitive function domains $\mathrm{T}$ scores, 5 points $(0.5$ SD) were adopted as distribution-based MCID. ${ }^{24}$ For the MoCA total score, 4 points were reported as the MDC in previous study. ${ }^{25}$ Statistical analyses were performed using $\mathrm{R}$ version $4.0 .2^{26}$ with a significance level of $5 \%$ and a two-sided test. 
Table 3 Baseline Demographic and Clinical Characteristics

\begin{tabular}{|c|c|c|c|c|}
\hline & & SHS $(n=6)$ & PMT $(n=5)$ & Total $(n=20)$ \\
\hline Age, y & & $70.0 \pm 6.9$ & $73.6 \pm 2.2$ & $71.0 \pm 5.1$ \\
\hline Gender, n (\%) & $\begin{array}{l}\text { Male } \\
\text { Female }\end{array}$ & $\begin{array}{l}\text { I (16.7\%) } \\
5 \text { (83.3\%) }\end{array}$ & $\begin{array}{l}\text { I (20.0\%) } \\
4(80.0 \%)\end{array}$ & $\begin{array}{c}3(15.0 \%) \\
17(85.0 \%)\end{array}$ \\
\hline Education & $\begin{array}{l}<6 \text { years } \\
\geq 6 \text { years }\end{array}$ & $\begin{array}{l}3(50.0 \%) \\
3(50.0 \%)\end{array}$ & $\begin{array}{l}2(40.0 \%) \\
3(60.0 \%)\end{array}$ & $\begin{array}{c}8(40.0 \%) \\
12(60.0 \%)\end{array}$ \\
\hline Employment & $\begin{array}{l}\text { Employed } \\
\text { Homemaker } \\
\text { Unemployed }\end{array}$ & $\begin{array}{l}2(33.3 \%) \\
2(33.3 \%) \\
2(33.3 \%)\end{array}$ & $\begin{array}{c}0(0.0 \%) \\
2(40.0 \%) \\
3(60.0 \%)\end{array}$ & $\begin{array}{l}6(30.0 \%) \\
8(40.0 \%) \\
6(30.0 \%)\end{array}$ \\
\hline Medical history & $\begin{array}{l}\text { Hypertension } \\
\text { Diabetes mellitus } \\
\text { Hyperlipidemia }\end{array}$ & $\begin{array}{l}4(66.7 \%) \\
\text { I (16.7\%) } \\
0(0.0 \%)\end{array}$ & $\begin{array}{l}4(80.0 \%) \\
2(40.0 \%) \\
2(40.0 \%)\end{array}$ & $\begin{array}{l}14(70.0 \%) \\
7(35.0 \%) \\
4(20.0 \%)\end{array}$ \\
\hline BMI & & $24.6 \pm 1.8$ & $23.4 \pm 3.1$ & $25.2 \pm 2.9$ \\
\hline $\mathrm{MCl}$ subtypes & $\begin{array}{l}\text { aMCl (single) } \\
\text { aMCl (multiple) } \\
\text { naMCl (single) } \\
\text { naMCl (multiple) } \\
\text { Unspecified }\end{array}$ & $\begin{array}{c}0(0.0 \%) \\
1(16.7 \%) \\
0(0.0 \%) \\
3(50.0 \%) \\
2(33.3 \%)\end{array}$ & $\begin{array}{l}\text { I }(20.0 \%) \\
\text { I }(20.0 \%) \\
\text { I }(20.0 \%) \\
\text { I }(20.0 \%) \\
\text { I }(20.0 \%)\end{array}$ & $\begin{array}{l}3(15.0 \%) \\
6(30.0 \%) \\
3(15.0 \%) \\
4(20.0 \%) \\
4(20.0 \%)\end{array}$ \\
\hline CDR-SB (0-18) & & $3.2 \pm 0.4$ & $3.3 \pm 0.4$ & $3.2 \pm 0.3$ \\
\hline GDS & $\begin{array}{l}\text { GDS } 2 \\
\text { GDS } 3 \\
\text { GDS } 4\end{array}$ & $\begin{array}{c}5(83.3 \%) \\
0(0.0 \%) \\
I(16.7 \%)\end{array}$ & $\begin{array}{c}4(80.0 \%) \\
0(0.0 \%) \\
I(20.0 \%)\end{array}$ & $\begin{array}{l}\text { I6 (80.0\%) } \\
\text { I (5.0\%) } \\
3(15.0 \%)\end{array}$ \\
\hline $\operatorname{MoCA}(0-30)$ & & $18.0 \pm 2.6$ & $18.4 \pm 3.0$ & $17.6 \pm 4.2$ \\
\hline SNSB-II five domains T score & $\begin{array}{l}\text { Attention } \\
\text { Language } \\
\text { Visuospatial } \\
\text { Memory } \\
\text { Frontal/executive }\end{array}$ & $\begin{array}{c}45.9 \pm 7.0 \\
43.9 \pm 13.0 \\
38.2 \pm 11.9 \\
43.5 \pm 10.8 \\
45.1 \pm 11.1\end{array}$ & $\begin{array}{c}52.0 \pm 12.1 \\
41.8 \pm 10.3 \\
47.7 \pm 11.9 \\
40.1 \pm 12.5 \\
49.0 \pm 8.3\end{array}$ & $\begin{array}{l}47.4 \pm 10.3 \\
37.8 \pm 17.3 \\
39.8 \pm 19.3 \\
39.7 \pm 12.7 \\
42.9 \pm 18.5\end{array}$ \\
\hline HIS $(0-18)$ & & $0.7 \pm 0.8$ & $1.8 \pm 1.1$ & $1.0 \pm 0.9$ \\
\hline $\begin{array}{l}\text { SGDepS }(0-15) \\
\text { KM Pattern score }(0-20)\end{array}$ & $\begin{array}{l}\text { Qi-deficiency } \\
\text { Yin-deficiency } \\
\text { Phlegm-dampness } \\
\text { Fire-heat }\end{array}$ & $\begin{array}{c}9.8 \pm 6.0 \\
12.3 \pm 6.2 \\
8.3 \pm 4.2 \\
9.5 \pm 5.3 \\
11.5 \pm 4.2\end{array}$ & $\begin{array}{l}6.6 \pm 6.4 \\
5.8 \pm 3.8 \\
5.2 \pm 3.1 \\
2.6 \pm 3.6 \\
3.2 \pm 2.5\end{array}$ & $\begin{array}{l}6.5 \pm 5.2 \\
8.8 \pm 6.3 \\
6.0 \pm 3.8 \\
5.8 \pm 5.2 \\
7.0 \pm 4.4\end{array}$ \\
\hline
\end{tabular}

Notes: Data are presented in mead \pm standard deviation or frequency $(\%)$.

Abbreviations: SHS, Samhwangsasim-tang; PMT, Palmul-tang; BMI, body mass index; $\mathrm{MCl}$, mild cognitive impairment; aMCl, amnestic mild cognitive impairment; naMCl, non-amnestic mild cognitive impairment; CDR-SB, Clinical Dementia Rating-Sum of Boxes; GDS, Global Deterioration Scale; MoCA, Montreal Cognitive Assessment; SNSBII, Seoul neuropsychological screening battery II; HIS, Hachinski's Ischemia Score; SGDepS, short version of geriatric depression scale.

\section{Results}

\section{Baseline Demographic and Clinical Characteristics}

Baseline demographic and clinical characteristics of total enrolled patients and patients who were prescribed SHS and PMT are presented in Table 3. One patient had mild dementia and 19 patients had MCI. Baseline demographic and clinical characteristics of patients who were prescribed YMJ, BHS, and YGJ are presented in Table S2. Of the 20 patients, the mean age was $71.0 \pm 5.1 \mathrm{y}$, and $85.0 \%$ were 
Table 4 SNSB-II Five Cognitive Function Domains T Score, and SGDepS Before and After the Treatment

\begin{tabular}{|c|c|c|c|c|}
\hline & Week $0(n=\mid 2)$ & Week I $2(n=12)$ & Week $24(n=I I)$ & $p$-value \\
\hline \multicolumn{5}{|c|}{ SNSB-II five cognitive domains $T$ score } \\
\hline $\begin{array}{l}\text { Attention } \\
\text { Difference } \\
\text { p-value }\end{array}$ & $50.79 \pm 8.84$ & $\begin{array}{c}56.14 \pm 8.84 \\
5.34(0.29,10.40) \\
0.0947\end{array}$ & $\begin{array}{c}52.36 \pm 9.08 \\
\mathrm{I} .56(-3.65,6.77) \\
0.8090\end{array}$ & 0.0785 \\
\hline $\begin{array}{l}\text { Language } \\
\text { Difference } \\
\text { p-value }\end{array}$ & $43.09 \pm 9.46$ & $\begin{array}{c}53.36 \pm 9.46 \\
10.27(2.40,18.14) \\
0.0333\end{array}$ & $\begin{array}{c}57.37 \pm 9.88 \\
14.28(6.23,22.33) \\
0.0037\end{array}$ & 0.0006 \\
\hline $\begin{array}{c}\text { Visuospatial } \\
\text { Difference } \\
\text { p-value }\end{array}$ & $45.22 \pm 9.27$ & $\begin{array}{c}55.06 \pm 9.27 \\
9.84(3.19,16.48) \\
0.0150\end{array}$ & $\begin{array}{c}54.91 \pm 9.63 \\
9.69(2.86,16.51) \\
0.0200\end{array}$ & 0.0022 \\
\hline $\begin{array}{l}\text { Memory } \\
\text { Difference } \\
\text { p-value }\end{array}$ & $40.93 \pm 11.52$ & $\begin{array}{c}51.74 \pm 11.52 \\
10.80(5.30,16.30) \\
0.0015\end{array}$ & $\begin{array}{c}56.61 \pm 1 \mid .75 \\
\mid 5.68(10.00,21.35) \\
<0.000 \mid\end{array}$ & $<0.0001$ \\
\hline $\begin{array}{l}\text { Frontal \& Executive } \\
\text { Difference } \\
p \text {-value }\end{array}$ & $48.15 \pm 8.36$ & $\begin{array}{c}52.74 \pm 8.36 \\
4.59(0.58,8.59) \\
0.0662\end{array}$ & $\begin{array}{c}53.98 \pm 8.53 \\
5.82(1.69,9.95) \\
0.0208\end{array}$ & 0.0074 \\
\hline \multicolumn{5}{|l|}{ Other indexes } \\
\hline $\begin{array}{l}\text { SGDepS (0-15) } \\
\text { Difference } \\
\text { p-value }\end{array}$ & $7.25 \pm 5.74$ & $\begin{array}{c}5.75 \pm 5.74 \\
-1.50(-2.87,-0.13) \\
0.0822\end{array}$ & $\begin{array}{c}6.84 \pm 5.77 \\
-0.4 I(-1.83, I .01) \\
0.8187\end{array}$ & 0.0645 \\
\hline
\end{tabular}

Notes: Data are presented in mead \pm standard deviation or mean $(95 \% \mathrm{Cl})$. Least-square means and mean differences were calculated by the linear mixed model for the repeated measure. Pairwise comparison p-values were adjusted by Tukey's method.

Abbreviations: SNSB-II, Seoul neuropsychological screening battery II; SGDepS, short version of geriatric depression scale.

female. The KM pattern score of fire-heat was higher among patients who were prescribed SHS. Two patients who were prescribed YGJ displayed higher KM pattern scores in qi-deficiency and phlegm-dampness.

\section{Clinical Outcomes}

Among the five cognitive function domains of SNSB-II, language, visuospatial function, memory, and frontal/ executive function domains improved following herbal medicine treatment compared to baseline (Table 4). Considering the mean changes from the baseline in these three domains, $\mathrm{T}$ scores were larger than 5 points (0.5 SD) indicating MCID. Specifically, memory domain $\mathrm{T}$ score increased from $40.93 \pm 11.52$ to $51.74 \pm 11.52$ after 12 weeks (mean difference: 10.80 [95\% CI: 5.30, $16.30], \mathrm{p}=0.0015$ ), and to $56.61 \pm 11.75$ after 24 weeks (mean difference: 15.68 [95\% CI: 10.00, 21.35], $\mathrm{p}<0.0001)$. The scores for cognitive tests included in each cognitive function domain generally improved after the treatment (Table S3). SGDepS decreased; however, this trend was not statistically significant. Subgroup analyses were performed for patients who were administered SHS or PMT and displayed tendencies similar to the results for all patients (Table S4 for SST, Table $\underline{\text { S5 }}$ for PMT).

The MoCA score gradually improved after the treatment over time (Table 5 and Figure 2). At the week-12 follow-up, the MoCA score increased from $17.73 \pm 5.28$ to $21.96 \pm 5.42$ (mean difference: 4.23 [95\% CI: 2.60 , $5.86], \mathrm{p}<0.0001)$. The mean change from the baseline in MoCA score was larger than 4 points which corresponded to MDC. The result of the KM pattern scores is also presented in Table 5. There was no consistent tendency according to time in the scores for qideficiency or yin-deficiency. Scores for phlegmdampness and fire-heat tended to decrease over time after the treatment; however, this trend was not statistically significant. Patients' satisfaction with the treatment was measured by the IMPSS at weeks 12 and 24 (Table 6); $50 \%$ and $64 \%$ of the patients answered that they were satisfied or very satisfied with the herbal medicine treatment, respectively. 
Table 5 Montreal Cognitive Assessment (MoCA) and Korean Medicine (KM) Pattern Scores Before and After the Treatment

\begin{tabular}{|c|c|c|c|c|c|}
\hline & Week $0(n=15)$ & Week $6(n=15)$ & Week I $2(n=12)$ & Week $24(n=I I)$ & p-value \\
\hline \multicolumn{6}{|l|}{ MoCA } \\
\hline $\begin{array}{l}\text { MoCA } \\
\text { Difference } \\
\text { p-value }\end{array}$ & $17.73 \pm 5.28$ & $\begin{array}{c}21.40 \pm 5.28 \\
3.67(2.17,5.17) \\
0.0001\end{array}$ & $\begin{array}{c}21.96 \pm 5.42 \\
4.23(2.60,5.86) \\
<0.0001\end{array}$ & $\begin{array}{c}24.03 \pm 5.48 \\
6.29(4.61,7.97) \\
<0.0001\end{array}$ & $<0.0001$ \\
\hline \multicolumn{6}{|l|}{ KM pattern scores } \\
\hline $\begin{array}{l}\text { Qi-deficiency } \\
\text { Difference } \\
\text { p-value }\end{array}$ & $9.67 \pm 6.09$ & $\begin{array}{c}10.13 \pm 6.09 \\
0.47(-1.60,2.53) \\
0.9676\end{array}$ & $\begin{array}{c}9.43 \pm 6.32 \\
-0.24(-2.49,2.01) \\
0.9963\end{array}$ & $\begin{array}{c}8.85 \pm 6.41 \\
-0.81(-3.13,1.50) \\
0.8915\end{array}$ & 0.7319 \\
\hline $\begin{array}{l}\text { Yin-deficiency } \\
\text { Difference } \\
\text { p-value }\end{array}$ & $7.00 \pm 3.99$ & $\begin{array}{c}5.67 \pm 3.99 \\
-1.33(-3.28,0.62) \\
0.5156\end{array}$ & $\begin{array}{c}7.62 \pm 4.27 \\
0.62(-1.49,2.73) \\
0.9337\end{array}$ & $\begin{array}{c}5.99 \pm 4.39 \\
-1.01(-3.18,1.17) \\
0.7839\end{array}$ & 0.2087 \\
\hline $\begin{array}{l}\text { Phlegm-dampness } \\
\text { Difference } \\
\text { p-value }\end{array}$ & $6.60 \pm 5.02$ & $\begin{array}{c}6.53 \pm 5.02 \\
-0.07(-2.27,2.14) \\
0.9999\end{array}$ & $\begin{array}{c}5.75 \pm 5.32 \\
-0.85(-3.23, \quad \text { I.54) } \\
0.889 \text { I }\end{array}$ & $\begin{array}{c}3.88 \pm 5.44 \\
-2.72(-5.18,-0.26) \\
0.1321\end{array}$ & 0.1053 \\
\hline $\begin{array}{c}\text { Fire-heat } \\
\text { Difference } \\
\text { p-value }\end{array}$ & $8.07 \pm 4.74$ & $\begin{array}{c}8.07 \pm 4.74 \\
0.00(-1.52, \quad 1.52) \\
0.9999\end{array}$ & $\begin{array}{c}7.94 \pm 4.90 \\
-0.13(-1.79,1.53) \\
0.9985\end{array}$ & $\begin{array}{c}6.02 \pm 4.96 \\
-2.04(-3.75,-0.34) \\
0.0902\end{array}$ & 0.0487 \\
\hline
\end{tabular}

Notes: Data are presented in mead \pm standard deviation or mean $(95 \% \mathrm{Cl})$. Least-square means and mean differences were calculated by the linear mixed model for the repeated measure. Pairwise comparison p-values were adjusted by Tukey's method.

\section{Safety Outcomes}

During the study period, five adverse events were reported from four patients. Two adverse events from one patient that were likely related to the interventions. A patient who was administered YMJ reported mild adverse events of nausea and chest discomfort, and both symptoms completely resolved naturally without further intervention. There were no other intervention-related adverse events reported by the remaining patients. No serious adverse event was observed in the study. The complete blood count, and liver and kidney function tests conducted at baseline and week 24 indicated no clinically significant changes (Table S6).

\section{Discussion}

\section{Herbal Medicine for Patients with Neurocognitive Disorders}

In this observational study of patients with neurocognitive disorders who planned to be treated with herbal medicine, SHS (30\%) and PMT (25\%) were frequently prescribed. Following herbal medicine treatment, the patients' cognitive functions improved at the 12 and 24 weeks' followups. In KM pattern scores, phlegm-dampness and fire-heat scores tended to improve after the treatment. Throughout the study, mild adverse events of nausea and chest discomfort were reported; however, no serious adverse events occurred.

Various herbal medicine formulae were used in this study according to the pattern identification of each patient. YMJ, which is consists of six crude herbs reinforcing yin-deficiency, was reported to ameliorate cognitive impairment in a mouse model. ${ }^{27}$ Additionally, YMJ showed effectiveness in improving dry mouth (xerostomia) a typical symptom of yin-deficiency, for older adult patients. ${ }^{28}$ SHS includes three crude herbs that clear heat; Rhei Radix et Rhizoma and Coptidis Rhizoma are also included in the herbal granule, GRAPE formula, which demonstrated significant benefit for patients with $\mathrm{AD} .{ }^{13}$ PMT is composed of herbs to enhance Qi and blood, and PMT-containing formula attenuated memory deficits in in vivo studies. ${ }^{29,30}$ Moreover, BHS prevented lipopolysaccharide-induced cognitive impairment and neuroinflammation in mice. ${ }^{31}$ The herbal medicine formulae used in this observational study demonstrated potential effects on cognitive impairment in previous animal and human studies.

In this study, we used licensed herbal medicine products manufactured by pharmaceutical companies that were produced according to the Korean Herbal Pharmacopoeia (KHP), and modification of compositional 


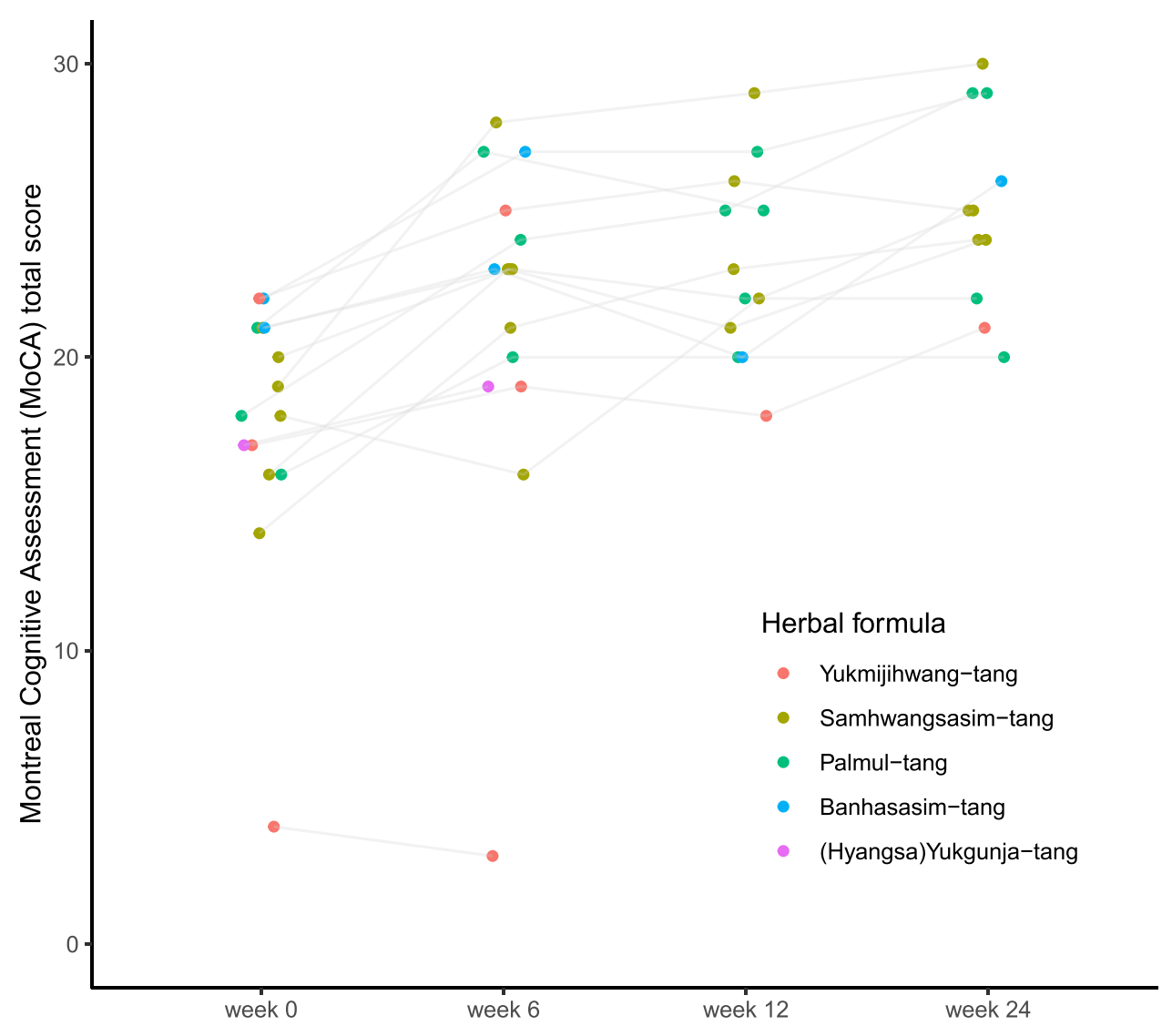

Figure 2 Montreal Cognitive Assessment (MoCA) score for patients over time.

herbs was not possible. Therefore, the use of frequently added herbs for cognitive disorders, such as Acori Graminei Rhizoma and Polygalae Radix ${ }^{32-34}$ was difficult. In this study, the patients' cognitive functions generally improved after the herbal medicine treatment, even without those herbs. Another similar study incorporated licensed herbal medicine products for patients with $\mathrm{MCI}^{35}$ and reported that older adults demonstrated improvement in MoCA score after six months of herbal medicine treatment. The modified Guibi-tang (Guipi-tang in Chinese, Kihi-to in Japanese) was the most frequently

Table 6 Integrative Medicine Patient Satisfaction Scale (IMPSS) After the Treatment

\begin{tabular}{|l|c|c|}
\hline & Week I2 (n= I 2) & Week 24 (n= I I) \\
\hline Very satisfied & $3(25.0 \%)$ & $2(18.5 \%)$ \\
Satisfied & $3(25.0 \%)$ & $5(45.5 \%)$ \\
Neutral & $6(50.0 \%)$ & $4(36.4 \%)$ \\
Dissatisfied & $0(0.0 \%)$ & $0(0.0 \%)$ \\
Very dissatisfied & $0(0.0 \%)$ & $0(0.0 \%)$ \\
\hline
\end{tabular}

Note: Data are presented in frequency (\%). used formula (48.9\%) in the study, and its composition and indication are similar to PMT.

\section{KM Patterns of Patients with Neurocognitive Disorders}

The KM patterns of the enrolled patients were determined by combining the results of PIT-C and clinicians' examination in this study. At the baseline assessment, six patients had a Fire-heat pattern (30\%), five patients had a Qi and blood-deficiency pattern (25\%), four patients had a Phlegmdampness pattern (20\%), three patients had a Yin-deficiency pattern (15\%), and two patients had Qi-deficiency pattern $(10 \%)$. In another study of TCM constitution in 152 patients with MCI, neutral was the most frequent constitution (33.6\%), followed by Qi-deficient (33.5\%), Yang-deficient (21.7\%), Phlegm-dampness (9.2\%), and Blood-stasis $(7.9 \%){ }^{12}$ In a study of 52 mild to moderate patients with $\mathrm{AD}$, liver-kidney yin deficiency was the most frequent pattern $(64.8 \%) .{ }^{36}$ In a randomized controlled trial of patients with mild to moderate $\mathrm{AD}$, appropriate herbal medicine was prescribed according to the pattern 
identification of Heart Qi-deficiency, Kidney Yindeficiency, Phlegm-dampness, and Blood stasis. ${ }^{37}$ In previous studies, patterns of Qi-deficiency, Yin-deficiency, Phlegm-dampness, and Blood stasis were commonly reported among patients with neurocognitive disorders.

After the 12 to 24 weeks of herbal medicine treatment, KM patterns of phlegm-dampness and fire-heat score improved, whereas qi-deficiency and yin-deficiency scores did not. The deficiency patterns may require longer treatment to improve compared to excess patterns, such as phlegm-dampness and fire-heat. In a previous study of patterns among patients with $\mathrm{AD}$, Shen (Kidney) deficiency was observed throughout all stages of AD. ${ }^{14}$ For patients with MCI, Qinggongshoutao, which is traditionally known to enhance kidney function, was administrated for 52 weeks in a randomized controlled trial. ${ }^{38,39}$ In that study, $67.2 \%$ of patients who were administrated herbal medicine showed improvement in deficiency of kidney essence, after the relatively long treatment period. In general, deficiency patterns are more commonly observed in chronic disease than acute disease. ${ }^{40-42}$ To observe the improvement in Qi-deficiency and Yin-deficiency pattern scores for patients with MCI, the administration period of herbal medicine probably needs to be longer than 12 weeks.

There are a few other studies that reported changes in TCM, KM pattern syndrome in patients with MCI. In a study of patients with MCI, TCM syndrome scores, including kidney essence deficiency, phlegm, and blood stasis were reduced after treatment with herbal medicine. ${ }^{43}$ Another herbal medicine formula, Huannao Yicong formula, also reported to improve the Chinese Medicine Symptom Scale (CM-SS) as well as cognitive function in patients with mild to moderate AD. ${ }^{44}$ The two previously mentioned studies incorporated only the sum of each TCM pattern score. To evaluate the effect of herbal medicine for patients with neurocognitive disorders, the development of common TCM or KM pattern scores is needed. There is limited information regarding the blood stasis pattern symptoms in PIT-C. ${ }^{15}$ It would be preferable if the revised pattern identification tool were capable of reflecting the stage of the cognitive disorders and contain essential patterns of cognitive disorders; Qi-deficiency, Yin-deficiency, Phlegm-dampness, Blood stasis, and Fire-Heat.

\section{Limitations and Interpretation}

There are several limitations to our study. First, it was an observational study without a control group. The effect of herbal medicine on neurocognitive impairment was measured by comparing the baseline and the post-treatment, which cannot exclude the possibility of the placebo effect and the influence of the learning effect. The results of this study is not sufficient to conclude the effect of herbal medicine on neurocognitive impairment. However, the mean change from the baseline after the treatment in MoCA showed improvement beyond the MCID and MDC, indicates the potential clinical effect of herbal medicine on cognitive function. Second, various formulae were used for patients, and the results cannot answer the question of which formulae, herbs, or components were effective for cognitive function improvement. Third, the sample size was relatively small. Nevertheless, cognitive function and $\mathrm{KM}$ pattern scores were observed before and after the herbal medicine treatment. For most patients with cognitive disorders, compliance on taking herbal medicine three times a day was higher than $70 \%$, and cognitive functions generally improved after the treatment. There was no clinically significant abnormal change observed after the treatment on assessment by the liver and kidney function tests. Additionally, we used licensed herbal medicine products in Korea, which are also available in China and Japan, and can be easily applied in the real-world clinical setting.

\section{Conclusion}

This study explored the effect of herbal medicine treatment according to the KM pattern identification for patients with MCI and early dementia. Application of the herbal medicine treatment approach used in this study could be considered a possible option with very few undesirable side-effects and potentially improve cognitive function in patients with MCI. Further research is needed to objectify the KM pattern identification process and evaluate the KM pattern-related signs and symptoms.

\section{Acknowledgments}

This research was supported by grants from Korea Institute of Oriental Medicine [KSN2021230]

\section{Disclosure}

The authors report no conflicts of interest in this work.

\section{References}

1. American Psychiatric Association. Diagnostic and Statistical Manual of Mental Disorders $\left(D S M-5^{\circledR}\right)$. American Psychiatric Pub; 2013. 
2. Sachdev PS, Blacker D, Blazer DG, et al. Classifying neurocognitive disorders: the DSM-5 approach. Nat Rev Neurol. 2014;10(11):634. doi:10.1038/nrneurol.2014.181

3. May BH, Lu C, Lu Y, Zhang AL, Xue CC. Chinese herbs for memory disorders: a review and systematic analysis of classical herbal literature. J Acupunct Meridian Stud. 2013;6(1):2-11. doi:10.1016/j. jams.2012.11.009

4. May BH, Yang AW, Zhang AL, et al. Chinese herbal medicine for mild cognitive impairment and age associated memory impairment: a review of randomised controlled trials. Biogerontology. 2009;10 (2):109-123. doi:10.1007/s10522-008-9163-5

5. Dong L, Hyde AJ, Zhang AL, Xue CC, May BH. Chinese herbal medicine for mild cognitive impairment using Montreal cognitive assessment: a systematic review. $J$ Altern Complement Med. 2019;25(6):578-592. doi:10.1089/acm.2018.0346

6. Hyde AJ, May BH, Dong L, et al. Herbal medicine for management of the behavioural and psychological symptoms of dementia (BPSD): a systematic review and meta-analysis. J Psychopharmacol. 2017;31 (2):169-183. doi:10.1177/0269881116675515

7. Kim KH, Go HY, Lee JA, et al. The effect of Dangguijagyag-san on mild cognitive impairment. J Altern Complement Med. 2016;22 (7):509-514. doi:10.1089/acm.2015.0261

8. Kudoh C, Arita R, Honda M, et al. Effect of ninjin'yoeito, a Kampo (traditional Japanese) medicine, on cognitive impairment and depression in patients with A lzheimer's disease: 2 years of observation. Psychogeriatrics. 2016;16(2):85-92. doi:10.1111/psyg.12125

9. Jiang M, Lu C, Zhang C, et al. Syndrome differentiation in modern research of traditional Chinese medicine. $J$ Ethnopharmacol. 2012;140(3):634-642. doi:10.1016/j.jep.2012.01.033

10. Birch S, Alraek T, Bovey M, et al. Overview on pattern identificationhistory, nature and strategies for treating patients: a narrative review. Eur J Integr Med. 2020;35:101101. doi:10.1016/j.eujim.2020.101101

11. Shi J, Tian J, Long Z, et al. The pattern element scale: a brief tool of traditional medical subtyping for dementia. Evid Based Complement Alternat Med. 2013;2013:1-10. doi:10.1155/2013/460562

12. Deng X, Teng J, Nong X, et al. Characteristics of TCM constitution and related biomarkers for mild cognitive impairment. Neuropsychiatr Dis Treat. 2021;17:1115. doi:10.2147/NDT.S290692

13. Shi J, Ni J, Lu T, et al. Adding Chinese herbal medicine to conventional therapy brings cognitive benefits to patients with Alzheimer's disease: a retrospective analysis. BMC Complement Altern Med. 2017;17(1):1-7. doi:10.1186/s12906-017-2040-5

14. Tian JZ, Shi J, Ni JN, et al. Sequential therapy based on evolvement of patterns: a new model for treatment of Alzheimer's disease. Chin J Integr Med. 2019;25(8):565-573. doi:10.1007/s11655-019-3066-y

15. Lee JY, Kim H, Seo YK, Kang HW, Kang WC, Jung IC. A research to evaluate the reliability and validity of pattern identifications tool for cognitive disorder: a clinical study protocol. $J$ Orient Neuropsychiatry. 2018;29(4):255-266.

16. Moon KS, Kim NK, Jung IC, Kang HW. Preliminary study to develop the Korean medical pathologic aging scale and Korean medical pattern identification for dementia. J Korean Med. 2017;38 (3):111-123. doi:10.13048/jkm.17030

17. Seo YK, Oh YC, Lee JJ, et al. Exploring the effective herbal prescription for cognitive disorder Treatment among licensed herbal medicines in Korea-A preliminary study for clinical trial of cognitive disorders. J Physiol Pathol Korean Med. 2019;33(4):207-218. doi:10.15188/kjopp.2019.08.33.4.207

18. Craft JM, Watterson DM, Van Eldik LJ. Neuroinflammation: a potential therapeutic target. Expert Opin Ther Targets. 2005;9 (5):887-900. doi:10.1517/14728222.9.5.887

19. Saxena U. Bioenergetics breakdown in Alzheimer's disease: targets for new therapies. Int J Physiol Pathophysiol Pharmacol. 2011;3 (2): 133 .
20. Rossi A, Rigotto G, Valente G, et al. Defective mitochondrial pyruvate flux affects cell bioenergetics in Alzheimer's disease-related models. Cell Rep. 2020;30(7):2332-2348. e2310. doi:10.1016/j. celrep.2020.01.060

21. Kang Y, Jang S, Na D Seoul neuropsychological screening battery (SNSB-II). Seoul, Korea: Human Brain Research \& Consulting Co; 2012.

22. Ahn HJ, Chin J, Park A, et al. Seoul Neuropsychological Screening Battery-dementia version (SNSB-D): a useful tool for assessing and monitoring cognitive impairments in dementia patients. $J$ Korean Med Sci. 2010;25(7):1071-1076. doi:10.3346/jkms.2010.25.7.1071

23. Steinsbekk A, Biolchini J, Heger M, Rezzani C, Tsamis N, van Haselen R. Data collection in homeopathic practice-A proposal for an international standard. HomInt RD NewsLetter. 1999;2:14-15.

24. Norman GR, Sloan JA, Wyrwich KW. Interpretation of changes in health-related quality of life: the remarkable universality of half a standard deviation. Med Care. 2003;41(5):582-592. doi:10.1097/ 01.MLR.0000062554.74615.4C

25. Feeney J, Savva GM, O’Regan C, King-Kallimanis B, Cronin H, Kenny RA. Measurement error, reliability, and minimum detectable change in the mini-mental state examination, Montreal cognitive assessment, and color trails test among community living middle-aged and older adults. $J$ Alzheimers Dis. 2016;53 (3):1107-1114. doi:10.3233/JAD-160248

26. R: a language and environment for statistical computing. $\mathrm{R}$ Foundation for Statistical Computing; 2020. Available from: https://www.R-project.org/. Accessed October 6, 2021.

27. Eom TM, Kwon HH, Shin N, et al. Traditional Korean herbal formulae, Yuk-Mi-Ji-Hwang-Tang, ameliorates impairment of hippocampal memory ability by chronic restraint stress of mouse model. J Ethnopharmacol. 2020;260:113102. doi:10.1016/j.jep.20 20.113102

28. Han G, Ko SJ, Kim J, Oh JY, Park JW, Kim J. A randomized, double-blind, placebo-controlled trial of a traditional herbal formula, Yukmijihwang-tang in elderly subjects with xerostomia. J Ethnopharmacol. 2016;182:160-169. doi:10.1016/j.jep.2016.02.014

29. Yun YJ, Lee B, Hahm DH, et al. Neuroprotective effect of palmul-chongmyeong-tang on ischemia-induced learning and memory deficits in the rat. Biol Pharm Bull. 2007;30(2):337-342. doi:10.1248/bpb.30.337

30. Kim YR, Kwon MY, Pak ME, Park SH, Baek JU, Choi BT Beneficial effects of gagam-palmultang on scopolamine-induced memory deficits in mice. Evid Based Complement Alternat Med. 2018;2018:1-11. doi:10.1155/2018/3479083

31. Oh YC, Jeong YH, Pak ME, Go Y. Banhasasim-tang attenuates lipopolysaccharide-induced cognitive impairment by suppressing neuroinflammation in mice. Nutrients. 2020;12(7):2019. doi:10. 3390/nu12072019

32. Choi Y, Kim YE, Jerng UM, et al. Korean traditional medicine in treating patients with mild cognitive impairment: a multicenter prospective observational case series. Evid Based Complement Alternat Med. 2020;2020:1-12. doi:10.1155/2020/4323989

33. May BH, Feng M, Zhou IW, et al. Memory impairment, dementia, and Alzheimer's disease in classical and contemporary traditional Chinese medicine. J Altern Complement Med. 2016;22(9):695-705. doi:10.1089/acm.2016.0070

34. May BH, Lu C, Bennett L, Hügel HM, Xue CC. Evaluating the traditional Chinese literature for herbal formulae and individual herbs used for age-related dementia and memory impairment. Biogerontology. 2012;13(3):299-312. doi:10.1007/s10522-012-9375-6

35. Kim YN, Bae JS, Eom YJ, Lee KS, Yoon HM, Cho SH. Results of Korean medicine treatment in community dwelling elderly with mild cognitive impairment: focusing on the change in cognitive ability. J Orient Neuropsychiatry. 2019;30(3):185-198. 
36. Kim KN, Bae H, Hwang WW, Cho SH. Study on the application of oriental medical evaluation to Dementia. J Orient Neuropsychiatry. 2014;25(4):383-388. doi:10.7231/jon.2014.25.4.383

37. Yu L, Lin S, Zhou R, et al. Chinese herbal medicine for patients with mild to moderate Alzheimer disease based on syndrome differentiation: a randomized controlled trial. Zhong Xi Yi Jie He Xue Bao. 2012;10(7):766-776.

38. Tian J, Shi J, Wei $M$, et al. Chinese herbal medicine Qinggongshoutao for the treatment of amnestic mild cognitive impairment: a 52-week randomized controlled trial. Alzheimers Dement. 2019;5(1):441-449.

39. Shi J, Ni J, Wei M, et al. Association between pattern changes and cognitive outcome in Alzheimer's disease. J Beijing Univ Tradit Chin Med. 2017;54:339-343.

40. Kim J, Ku B, Kim KH. Validation of the qi blood yin yang deficiency questionnaire on chronic fatigue. Chin Med. 2016;11(1):1-11. doi:10.1186/s13020-016-0092-y
41. Yakubo S, Ito M, Ueda $\mathrm{Y}$, et al. Pattern classification in Kampo medicine. Evid Based Complement Alternat Med. 2014;2014:1-5. doi:10.1155/2014/535146

42. Bae KH, Go HY, Park KH, Ahn I, Yoon Y, Lee S. The association between cold hypersensitivity in the hands and feet and chronic disease: results of a multicentre study. BMC Complement Altern Med. 2018;18(1):1-8. doi:10.1186/s12906-018-2082-3

43. Miao Y, Tian J, Shi J, Mao M. Effects of Chinese medicine for tonifying the kidney and resolving phlegm and blood stasis in treating patients with amnestic mild cognitive impairment: a randomized, double-blind and parallel-controlled trial. Zhong Xi Yi Jie He Xue Bao. 2012;10(4):390-397.

44. Yang Y, Liu JP, Fang JY, et al. Effect and safety of huannao yicong formula in patients with mild-to-moderate Alzheimer's disease: a randomized, double-blinded, donepezil-controlled trial. Chin J Integr Med. 2019;25(8):574-581. doi:10.1007/s11655-0183054-7

\section{Publish your work in this journal}

Neuropsychiatric Disease and Treatment is an international, peerreviewed journal of clinical therapeutics and pharmacology focusing on concise rapid reporting of clinical or pre-clinical studies on a range of neuropsychiatric and neurological disorders. This journal is indexed on PubMed Central, the 'PsycINFO' database and CAS, and is the official journal of The International Neuropsychiatric Association (INA). The manuscript management system is completely online and includes a very quick and fair peer-review system, which is all easy to use. Visit http://www.dovepress.com/testimonials.php to read real quotes from published authors. 